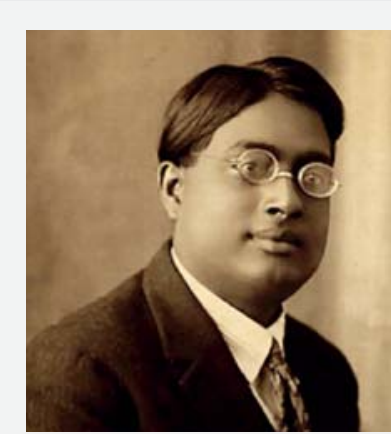

Principales dates

1er janvier 1894 - Naissance à Calcutta (Inde)

1924

1945

Découverte de la statistique de Bose

1958 Invention du terme Boson par Dirac

Nommé National Professor

4 février 1974 - Décès à Calcutta (Inde)

\title{
Satyendranath Bose
}

\section{Riad Haidar, haidar@onera.fr}

Scientifique indien, spécialiste de la physique mathématique, pionnier de l'enseignement et de la recherche de l'Inde post-coloniale, il est surtout connu pour avoir démontré la loi du rayonnement de Planck en inventant une nouvelle façon de dénombrer les particules indiscernables - la célèbre statistique de Bose. Ce travail a joué un rôle déterminant dans la gestation de la physique quantique moderne.

$\mathrm{S}$ atyendranath, que ses concitoyens bengalis surnomment

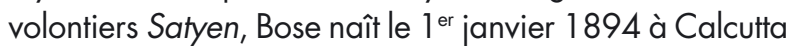
(ou Kolkata), qui est alors la capitale de l'empire britannique des Indes. Sa famille paternelle appartient à la caste de lettrés Kayastha, et est originaire de Bara Jajuli, un village prospère du district de Nadia, qui fut au moyen-âge un haut-lieu de la vie intellectuelle et culturelle du Bengale, à la frontière de l'Inde et du Bangladesh actuels. Son grand-père a été un employé de l'administration coloniale anglaise. Son père Surendranath Bose a travaillé un temps comme comptable, avant de rejoindre la société des chemins de fer des Indes Orientales; à la fin du $19^{\mathrm{e}}$ siècle, il crée sa propre entreprise de produits chimiques et pharmaceutiques. La mère de Satyen, Amodini Devi, est la fille de Motilal Roy Choudhury, un avocat réputé d'Alipore, qui est issu d'une lignée de zamindars (aristocrates et riches propriétaires terriens) à Gaihati.

Satyen Bose est l'aîné, et le seul garçon, des sept enfants d'Amodini et de Surendranath. Sa scolarité débute à l'âge de cinq ans, près de son domicile. Lorsque sa famille déménage à Goabagan, il fréquente la New Indian School. II est, à cette époque, un élève moyen. Puis il passe une année à la Hindu School, et c'est le déclic : sous la férule d'un professeur charismatique, son intérêt s'éveille brusquement pour les mathématiques et les sciences. En 1909, il intègre le fameux Presidency College de Calcutta avec des résultats brillants. Il y partage les bancs avec le futur astrophysicien Meghnad Saha, et étudie sous la direction de professeurs illustres, tels que Jagadish Chandra Bose ou Prafulla Chandra Ray, qui lui inculquent une très haute ambition scientifique. Bose est un élève hors normes, qui accède très vite au statut de légende vivante. À I'Université de Calcutta, il choisit de se spécialiser en mathématiques appliquées et décroche un bachelor of science en 1913, puis un master of science en 1915, avec des résultats aux examens qui restent - dit-on - inégalés à ce jour. Encore étudiant, il épouse Ushabati, la fille unique de Jogindra Nath Ghosh, un médecin réputé du quartier de kombulia tôla, à Calcutta. Ils auront sept enfants, dont deux décèderont très jeunes.

\section{Travaux de recherche}

Bose poursuit ses études en sciences, et obtient un poste d'enseignant à la très récente University College of Science and Technology (UCST) de Calcutta en 1917. Cette jeune institution cultive un esprit moderne, et dispose $\mathrm{d}^{\prime}$ une bibliothèque fournie où Bose peut avoir accès aux travaux et recherches scientifiques les plus récents, notamment ceux menés en Europe sur la théorie quantique et la relativité - des textes alors introuvables en Inde. Bose dévore le livre de Gibbs sur la mécanique statistique, et obtient $d^{\prime}$ Einstein la permission de traduire et publier ses travaux en Inde. Depuis 1918, il publie régulièrement des papiers avec son ami Saha, en physique théorique et en mathématiques.

En 1921, il rejoint le département de physique de la prestigieuse Université de Dacca (aujourd'hui, au Bangladesh), que l'on surnomme l'Oxford of the East, sur la recommendation du charismatique Sir Ashutosh Mukheriee, physicien amateur et vice-chancelier de I'UCST. Bose entreprend d'organiser les départements de recherche en accord avec le programme d'enseignement pour les niveaux bachelor et master en sciences. Lui-même prend en 
charge l'enseignement de l'électromagnétisme de Maxwell et de la thermodynamique.

\section{Statistique de Bose-Einstein}

En 1924, tandis qu'il prépare un cours sur la théorie du rayonnement et qu'il s'apprête à en illustrer les limites en commentant la catastrophe ultraviolette' ${ }^{1}$ Bose trouve une astuce qui permet de démontrer la loi de Planck : comme Einstein avant lui, il considère le rayonnement du corps noir comme un gaz de photons, mais (et c'est précisément là son apport décisif, celui qui fera entrer son nom dans I'histoire) il traite ces photons comme des particules indiscernables, ce qui le mène à établir une nouvelle loi de distribution statistique.

II met ses notes au clair dans un court article qu'il intitule Planck's Law and the Hypothesis of Light Quanta, qui est rien de moins que l'acte fondateur de la mécanique quantique statistique. Mais son article est refusé par l'éditeur. II décide alors de l'envoyer à Einstien en Allemagne, accompagné d'une lettre :

Respected Sir, I have ventured to send you the accompanying article for your perusal and opinion. I am anxious to know what you think of it... I do not know whether you still remember that somebody from Calcutta asked your permission to translate your papers on Relativity in English. You acceded to the request. The book has since been published. I was the one who translated your paper on Generalised Relativity.

Einstein perçoit immédiatement le potentiel du formalisme de Bose et l'importance de l'article qu'il tient dans ses mains. Il le traduit lui-même en allemand et le soumet en juillet 1924, au nom de Bose, au prestigieux Zeitschrift für Physik, avec pour commentaire : « La démonstration par Bose de la formule de Planck est pour moi un important pas en avant». On connaît la suite. Notons, en signe de la formidable créativité de cette époque, qu'Einstein reçoit de Paul Langevin, ce même été 1924, une copie du manuscrit de thèse dans lequel Louis de Broglie établit la nature ondulatoire de la matière...

\section{L'essor}

Cet article et le parrainage d'Einstein valent à Bose une notoriété immédiate. Aussi, l'université de Dacca accède volontiers à sa requête d'effectuer un séjour scientifique en Europe. II passe une année à Paris, où il travaille avec Langevin et de Broglie, puis il rend visite à Einstein à Berlin en octobre 1925.

À son retour à Dacca en 1926, auréolé de gloire, il est nommé professeur (alors qu'il n'a pas de doctorat !) et prend la tête du département de physique. Scientifique à l'esprit ouvert et pénétrant, il développe activement l'enseignement des sciences, et organise les laboratoires et les bibliothèques pour fonder un centre avancé de recherches sur les rayons $X$ et la spectroscopie optique. Sa renommée internationale se renforce. Consécration suprême, Paul Dirac introduit en 1945, lors d'une conférence au Palais de

'La théorie prévoit que le rayonnement émis par un corps chauffé est inversement proportionnel au carré de la longueur d'onde. la Découverte de Paris, le terme boson pour les particules qui, comme les photons et dont on sait aujourd'hui que le spin est entier, obéissent à la statistique de Bose-Einstein.

En 1946, lorsque la partition de I'Inde devient imminente, Bose quitte Dacca et ce qui deviendra le Bangladesh. Il est nommé à la prestigieuse chaire Khaira à l'université de Calcutta, un poste qu'il occupe jusqu'à sa retraite en 1956. Très volontariste, il encourage ses étudiants à construire leur propre équipement à partir des moyens disponibles localement, dans leur environnement proche. II est élu Président de I'Indian Physical Society et du National Institute of Science en 1949. En 1958 il est élu Fellow de la Royal Society de Londres et, honneur suprême en Inde, il est nommé au poste de National Professor : c'est une position qu'il conservera pendant trois mandats consécutifs, pour une durée totale de 15 ans. En janvier 1974, la nation tout entière célèbre ses 80 ans, en même temps que le jubilé de la découverte de sa loi statistique. Bose doit assister à d'innombrables réceptions, expositions et séminaires qui attirent des savants du monde entier. Ce marathon médiatico-scientifique épuise ses forces. II succombe à une attaque éclair de broncho-pneumonie, le 4 février 1974, à Calcutta.

\section{Ultima verbae}

On peut s'étonner que Bose, dont les travaux ont initié et inspiré tant de prix Nobel pour des recherches sur les bosons, sur la statistique de Bose-Einstein ou encore sur le condensat de Bose-Einstein, n'ait pas lui-même été distingué par l'Académie Nobel. Interrogé sur cet oubli, Bose avait pris coutume de répondre : «j'ai déjà reçu toute la reconnaissance que je mérite ». II est probable qu'il le pensait sincèrement. Eł de fait, si l'on y réfléchit, il lui a été donné de faire une grande découverte et de la consigner en un seul papier de 4 pages - une découverte d'une importance inouie, qui a eu d'innombrables retombées et qui a rendu son nom immortel. Peu d'hommes dans l'histoire des sciences peuvent se prévaloir d'une réussite comparable.

\section{Références}

[1] Santimay Chatterjee, Enakshi Chatteriee, "S. Bose », National Book Trust, India (1976)

[2] Jagdish Mehra, "S. Bose », Biographical Memoirs of Fellows of the Royal Society (1975)

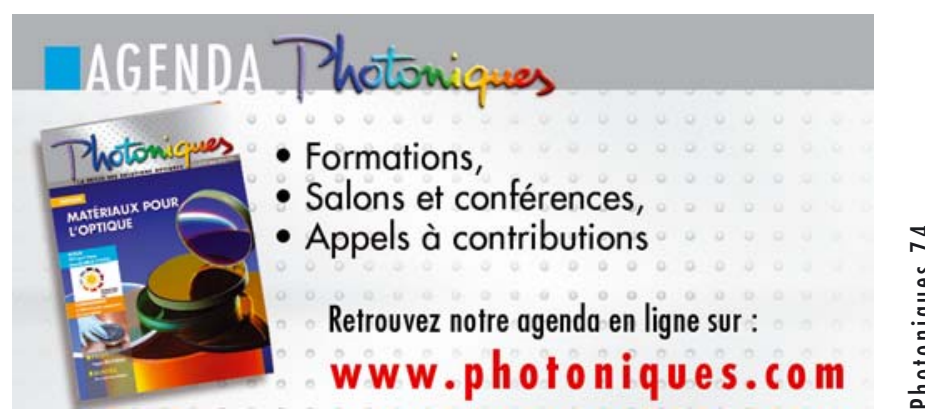

